\title{
Integrating SOM with K-Means in Taiwan TrainQuali System Performance Assessment
}

\author{
WenTsann Lin, ShihWei Shu \\ Dept. of Industrial Engineering and Management \\ National Chin-Yi University of Technology \\ Taichung City 41170, Taiwan
}

\author{
YungTsan Jou, Yihchuan $\mathrm{Wu}$ \\ Dept. of Industrial and System Engineering \\ Chung-Yuan Christian University \\ Chung-Li, Taoyuan County 32023, Taiwan \\ jason_wu1102@yahoo.com.tw
}

\begin{abstract}
The Taiwanese government has, based on the concept of the UK's Investors in People (IIP), and the Swiss ISO10015, drawn up the so-called Taiwan TrainQuali System (TTQS), a training process including Plan, Design, Do, Review, and Outcome. TTQS provides businesses and training institutions to carry out internal human training by offering a sound system of evaluating the education and training. In this study, confirmative comparison is made by two-stage clustering in "Self-Organizing Maps, SOM, and K-Means" to verify the consistence between the clustering results of all levels of grading. This study thus identifies the relevant factors that affect the effects of TTQS promotion, organizing and analyzing the current conditions regarding the extent of benefits for organizations from introducing the TTQS and the assistance to them in human training. Further, to verify, from the findings, that this training quality system can effectively enhance the human capital in organizations, such that the organizations that introduce and carry out TTQS all are able to construct the human training system that satisfies themselves.
\end{abstract}

Keywords- IIP; ISO10015; K-Means; SOM; TTQS

\section{INTRODUCTION}

The Bureau of Employment and Vocational Training (BEVT) set up the quality assurance system for human training in Taiwan that is relevant to the international training quality to uplift the human training quality and specialized ability as well as international competitive edge for Taiwan.

Starting in 2005, the TTQS specifications began to take shape with the efforts of many experts, aiming at the training of reviewers, developing grading criteria with TTQS score cards and completing the pretest of ten organizations. With the modification by the government, the present changes in foci on and objectives of TTQS development are apparent; for example, the foci on development up to 2009 were uplifting the quality of three types of persons, implementing the website specially for TTQS and integrating marketing and promotion, while the objectives of development were to establish review consistence and standardization, to strengthen digital network platform and to lay the mid-term training quality system plan. Through constant modifications, the government aims to make TTQS ensure of the effects of
Taiwanese enterprises' investment in human resources in a more complete manner.

\section{RESEARCH METHODS}

\section{A. TTQS Indicators}

Having a theme of investigating the performance in TTQS promotion, this study will use the 2010 TTQS Review database for analysis. In order to facilitate subsequent employment of related software to make analysis, this study will number the TTQS indicators. The indicators, bases for classification assessment and code list are as shown in Tables 1 below.

\section{B. Two-step Clustering}

Cluster analysis is a logical procedure of objective classification based on similarity and difference, having the purpose of facilitating the identification of the similarity between certain things and the division of them into a number of clusters by such feature such that those in a same cluster have high homogeneity and the samples among different clusters have high heterogeneity (Tzeng, 2010) [1].

Self-Organizing Map, SOM, by Kohonen (1990) [2], is applied to graphic recognition by mapping spatial data of high dimension to low dimension. As SOM makes clustering in the concept of nearest neighbor, the resultant clusters are seen with overlapping partitions. The resultant clusters of non-level-type clustering can produce nonoverlapping partitions. Abidi \& Ong (2000) [3] and Vesanto \& Alhoniemi (2000) [4] both proposed the technique of two-stage clustering as the clustering strategy for conducting data mining.

The algorithm of two-stage clustering is as follows:

1. SOM: Kohonen used self-organization to conduct graphic recognition, where vector values in consistence with the graphic elements are used to classify the graphs.

2. K-Means: in terms of economy, simplicity and effectiveness, K-Means is a method worth application. It is a non-layer-type clustering, not subject to the effects of outliers, errors in distance measurement and selection of method of distance calculation. The results of cluster are better if the initial point of the cluster is known. The knowledge of number of clusters ahead of making clusters enables effective process of large amount of numeric data; 
this method is suitable for dealing with the characteristics of convex clusters, while ending only fits local optimization.

TABLE I. BASES FOR CLASSIFICATION EVALUATION

\begin{tabular}{|c|c|c|c|}
\hline Code & Level & Performance level & Executive results \\
\hline 8 & $\begin{array}{l}\text { Platinum } \\
\text { medal }\end{array}$ & Benchmarking & $\begin{array}{l}\text { Eligible for example of success in the } \\
\text { industry }\end{array}$ \\
\hline 7 & Gold medal & Excellent & $\begin{array}{l}\text { Has really carried out and should } \\
\text { continue to improve }\end{array}$ \\
\hline 6 & Silver medal & Fair & $\begin{array}{l}\text { Has carried out as appropriate and } \\
\text { should make critical improvements }\end{array}$ \\
\hline 5 & Bronze medal & $\begin{array}{c}\text { A little } \\
\text { disastisfacory }\end{array}$ & $\begin{array}{l}\text { Has carried out partially and should } \\
\text { make major improvements }\end{array}$ \\
\hline 4 & Threshold & Poor & $\begin{array}{l}\text { Obvious evidence to executive results } \\
\text { has yet to be seen }\end{array}$ \\
\hline
\end{tabular}

\section{EMPIRICAL MEASUREMENT AND ANALYSIS}

This study uses all the businesses and training institutions in Taiwan (2010) [5] that have applied for "TTQS review service" to introduce the train quality system as research subjects.

After the analysis by Back-propagation neural networks model [6], comparison to verify is made by using two-stage clustering, which is to divide the data into a number of clusters such that data in a same cluster have high similarity; also, verification is conducted in the review results by the TTQS reviewers to compare with the reviews in the database for consistence. As such, the initial solutions obtained from the non-supervised SOM network in the first stage can be rendered into Table 2, the distribution of numbers of composing SOM clusters. These are further plotted into below Fig. 1. In the drawing for the distribution of SOM clusters, being the numbers of initial clusters in the first stage, the numbers are 9 in both "Business version" and "Training institution version".

To know the correct numbers of records in each cluster, it is possible to verify from the "distribution chart" the correct numbers of records in each cluster and the proportion of each cluster to the whole. Sorting results in Table 3, which indicates the distribution of composing numbers of K-Means clusters. As this study observes by "color normalization" with each cluster containing the records in the number of 100 , that includes the ratio of the number of fields selected by overlapping; hence, normalization allows better understanding when observing the relationship between the clusters and the set fields by overlapping.

Now the initial numbers of clusters are substituted into $\mathrm{K}$-Means before making step-two clustering of the nodes in the mapping drawing with K-Means of different concept of distances. A sorting results in Table 4 and Table 5, which is further plotted into Fig. 2, the distribution charts of twostage clustering model by SOM - K-Means, in which the Xaxis represents the initial numbers of clusters from step-one SOM and the $\mathrm{Y}$-axis represents the numbers of clusters of the initial numbers of clusters when substituted into KMeans. Now diagonal plotting is made to display the distribution chart for inspecting the status of the two-stage clustering, whereby (nine) can be determined as the number of clusters by two-stage clustering in the distribution chart of SOM - K-Means clusters in "Business version" and (nine) in "Training institution version". Consequently, the approximate number of records contained in each cluster can be obtained.

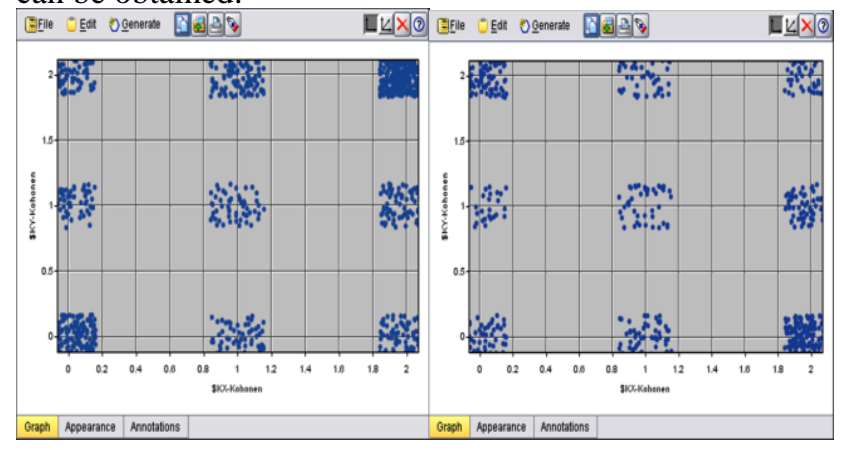

Fig. 1 SOM Clusters-Business version and Training institution version

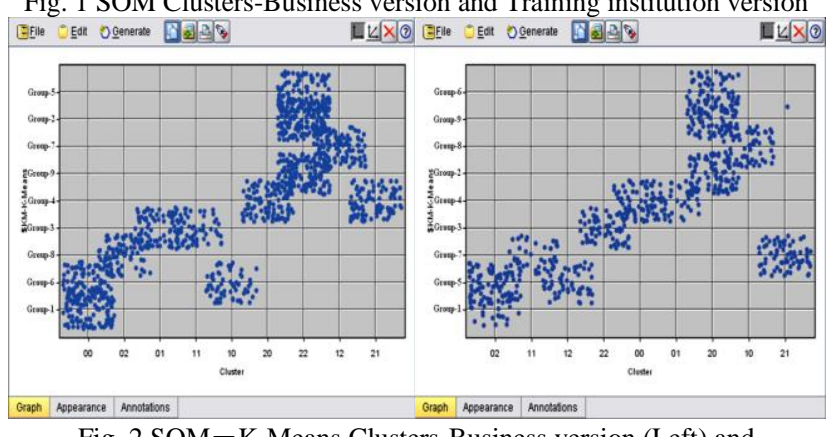

Fig. 2 SOM-K-Means Clusters-Business version (Left) and Training institution version (Right)

Fig. 2 above reveal the distribution of the distribution chart obtained by SOM-K-Means and further allows the observation of the degree of consistence. The analytical results in "Business version" are described as follows:

In respect to SOM clusters " 00,02 and 01 " and KMeans "Clusters 1, 6 and 8" falling in this area, because their composition includes "Gold" (22 organizations) which all fall in this area, it means the high consistence of the grading for the level of "Gold" by the reviewers. It also suggests that organizations under review who fall in this area have higher performance in TTQS promotion, with the indication that the organizations under review with "Silver", "Bronze" and "Threshold" that fall in this area all have higher levels of performance in execution than the corresponding grades and that their performances are higher than those of all the businesses who exercise TTQS. 
TABLE II. COMPOSITION TABLE OF SOM CLUSTERS

\begin{tabular}{|c|c|c|c|c|c|c|c|c|c|c|c|}
\hline \multicolumn{6}{|c|}{ Business version } & \multicolumn{6}{|c|}{ Training institution version } \\
\hline $\begin{array}{l}\$ K X \\
-K o h\end{array}$ & $\begin{array}{c}\$ K Y \\
-K o h\end{array}$ & Grade & Quant. & $\%$ & Total & $\begin{array}{r}\$ K X \\
-K o h\end{array}$ & $\begin{array}{c}\$ K Y \\
-K o h\end{array}$ & Grade & Quant. & $\%$ & Total \\
\hline 0 & 0 & Gold & 22 & 11.58 & \multirow{3}{*}{190} & 0 & 0 & Bronze & 26 & 36.62 & \multirow{2}{*}{71} \\
\hline 0 & 0 & Silver & 88 & 46.32 & & 0 & 0 & Threshold & 45 & 63.38 & \\
\hline 0 & 0 & Bronze & 80 & 42.11 & & 0 & 1 & Bronze & 39 & 100 & 39 \\
\hline 0 & 1 & Bronze & 82 & 100 & 82 & 0 & 2 & Gold & 12 & 11.11 & \multirow{3}{*}{108} \\
\hline 0 & 2 & Bronze & 30 & 34.09 & \multirow{2}{*}{88} & 0 & 2 & Silver & 36 & 33.33 & \\
\hline 0 & 2 & Threshold & 58 & 65.91 & & 0 & 2 & Bronze & 60 & 55.56 & \\
\hline 1 & 0 & Bronze & 62 & 100 & 62 & 1 & 0 & Threshold & 59 & 100 & 59 \\
\hline 1 & 1 & Bronze & 28 & 49.12 & \multirow{2}{*}{57} & 1 & 1 & Bronze & 28 & 63.64 & \multirow{2}{*}{44} \\
\hline 1 & 1 & Threshold & 29 & 50.88 & & 1 & 1 & Threshold & 16 & 36.36 & \\
\hline 1 & 2 & Threshold & 105 & 100 & 105 & 1 & 2 & Bronze & 52 & 100 & 52 \\
\hline 2 & 0 & Bronze & 61 & 58.65 & \multirow{2}{*}{104} & 2 & 0 & Threshold & 225 & 100 & 225 \\
\hline 2 & 0 & Threshold & 43 & 41.35 & & 2 & 1 & Threshold & 72 & 100 & 72 \\
\hline 2 & 1 & Threshold & 95 & 100 & 95 & 2 & 2 & Bronze & 46 & 65.71 & \multirow{2}{*}{70} \\
\hline 2 & 2 & Threshold & 404 & 100 & 404 & 2 & 2 & Threshold & 24 & 34.29 & \\
\hline
\end{tabular}

TABLE III. COMPOSITION TABLE OF K-MEANS CLUSTERS

\begin{tabular}{|c|c|c|c|c|c|c|c|c|c|c|c|}
\hline \multicolumn{6}{|c|}{ Business version } & \multicolumn{6}{|c|}{ Training institution version } \\
\hline Cluster & Code & Grade & Quant. & $\%$ & Total & Cluster & Code & Grade & Quant. & $\%$ & Total \\
\hline 1 & 7 & Gold & 22 & 23.16 & \multirow{3}{*}{95} & 1 & 7 & Gold & 12 & 40.00 & \multirow{2}{*}{30} \\
\hline 1 & 6 & Silver & 72 & 75.79 & & 1 & 6 & Silver & 18 & 60.00 & \\
\hline 1 & 5 & Bronze & 1 & 1.05 & & & & & & & \\
\hline 2 & 4 & Threshold & 157 & 100 & 157 & 2 & 4 & Threshold & 86 & 100 & 86 \\
\hline 3 & 5 & Bronze & 110 & 79.14 & \multirow{2}{*}{139} & 3 & 5 & Bronze & 46 & 65.71 & \multirow{2}{*}{70} \\
\hline 3 & 4 & Threshold & 29 & 20.86 & & 3 & 4 & Threshold & 24 & 34.29 & \\
\hline 4 & 5 & Bronze & 61 & 30.65 & \multirow{2}{*}{199} & 4 & 5 & Bronze & 65 & 59.09 & \multirow{2}{*}{110} \\
\hline 4 & 4 & Threshold & 138 & 69.35 & & 4 & 4 & Threshold & 45 & 40.91 & \\
\hline \multirow{2}{*}{5} & \multirow{2}{*}{4} & \multirow{2}{*}{ Threshold } & \multirow{2}{*}{107} & \multirow{2}{*}{100} & \multirow{2}{*}{107} & 5 & 6 & Silver & 18 & 13.85 & \multirow{2}{*}{130} \\
\hline & & & & & & 5 & 5 & Bronze & 112 & 86.15 & \\
\hline 6 & 6 & Silver & 16 & 10.19 & \multirow{2}{*}{157} & \multirow{2}{*}{6} & \multirow{2}{*}{4} & \multirow{2}{*}{ Threshold } & \multirow{2}{*}{61} & \multirow{2}{*}{100} & \multirow{2}{*}{61} \\
\hline 6 & 5 & Bronze & 141 & 89.81 & & & & & & & \\
\hline \multirow{2}{*}{7} & \multirow{2}{*}{4} & \multirow{2}{*}{ Threshold } & \multirow{2}{*}{105} & \multirow{2}{*}{100} & \multirow{2}{*}{105} & 7 & 5 & Bronze & 28 & 24.35 & \multirow{2}{*}{115} \\
\hline & & & & & & 7 & 4 & Threshold & 87 & 75.65 & \\
\hline 8 & 5 & Bronze & 30 & 34.09 & \multirow{2}{*}{88} & \multirow{2}{*}{8} & \multirow{2}{*}{4} & \multirow{2}{*}{ Threshold } & \multirow{2}{*}{59} & 100 & 50 \\
\hline 8 & 4 & Threshold & 58 & 65.91 & & & & & & 100 & 59 \\
\hline 9 & 4 & Threshold & 140 & 100 & 140 & 9 & 4 & Threshold & 79 & 100 & 79 \\
\hline
\end{tabular}

2. In respect to SOM clusters " 11,10 and 20 " and KMeans "Clusters 3, 4 and 9" falling in this area, because the composition of this area includes "Bronze" and "Threshold", it means that the organizations under review who fall in this area are seen to be inferior to those with "Gold" and "Silver" in terms of the performance in the operations of TTQS promotion, such as the extent to which indicators are carried out and the comprehensiveness, but they are not inferior to most organizations under review with "Threshold"; they thus present performance in TTQS promotion equal to the overall performance by all organizations.

3. In respect of the SOM clusters " 22,12 and 21" and K-Means "Clusters 7,2 and 5" falling in this area, because the composition in this area is solely "Threshold", it suggests that the organizations under review who fall in this area are relatively weak in the cognition and promotion of TTQS such that their levels of performance are lower than that by all TTQS promoting organizations. 
TABLE IV COMPOSING TABLE OF SOM - K-MEANS CLUSTERS

\begin{tabular}{|c|c|c|c|c|c|}
\hline \multicolumn{6}{|c|}{ Business version } \\
\hline SOM cluster & K-Means cluster & Grade & Quantity & $\%$ & Total \\
\hline 00 & Cluster 1 & Gold & 22 & $23.16 \%$ & \\
\hline 00 & Cluster 1 & Silver & 72 & $75.79 \%$ & 95 \\
\hline 00 & Cluster 1 & Bronze & 1 & $1.05 \%$ & \\
\hline 22 & Cluster 2 & Threshold & 157 & $100 \%$ & 157 \\
\hline 01 & Cluster 3 & Bronze & 82 & $59.00 \%$ & \\
\hline 11 & Cluster 3 & Bronze & 28 & $20.14 \%$ & 139 \\
\hline 11 & Cluster 3 & Threshold & 29 & $20.86 \%$ & \\
\hline 20 & Cluster 4 & Bronze & 61 & $30.65 \%$ & \\
\hline 20 & Cluster 4 & Threshold & 43 & $21.61 \%$ & 199 \\
\hline 21 & Cluster 4 & Threshold & 95 & $47.74 \%$ & \\
\hline 22 & Cluster 5 & Threshold & 107 & $100.00 \%$ & 107 \\
\hline 00 & Cluster 6 & Silver & 16 & $6.35 \%$ & \\
\hline 00 & Cluster 6 & Bronze & 79 & $31.35 \%$ & 252 \\
\hline 10 & Cluster 6 & Bronze & 157 & $62.30 \%$ & \\
\hline 12 & Cluster 7 & Threshold & 105 & $100.00 \%$ & 105 \\
\hline 02 & Cluster 8 & Bronze & 30 & $34.09 \%$ & \\
\hline 02 & Cluster 8 & Threshold & 58 & $65.91 \%$ & 88 \\
\hline 22 & Cluster 9 & Threshold & 140 & $100.00 \%$ & 140 \\
\hline
\end{tabular}

The analytic results in "Training institution version" are described as follows:

1. In respect of the SOM clusters " 02,11 and 12 " and K-Means "Clusters 1, 5 and 7" who fall in this area, because the composition of this area includes "Gold" (12 organizations), all of them falling in this area, it suggests high consistence of the grading for the level of "Gold" by the reviewers, also indicating that the organizations under review with "Silver", "Bronze" and "Threshold" that fall in this area all have higher levels of performance in execution than the corresponding grades and that their performances are higher than those of all the businesses who exercise TTQS

2. In respect of the SOM clusters " 22,00 and 01 " and K-Means "Clusters 3, 4 and 2" who fall in this area, because the composition of this area includes "Bronze" and "Threshold", the organizations under review who fall in this area are seen to be inferior to those with "Gold" and "Silver" in terms of the performance in the operations of TTQS promotion, such as the extent to which indicators are carried out and the comprehensiveness, but they are not inferior to most organizations under review with "Threshold"; they thus exhibit performance in TTQS promotion equal to the overall performance by all organizations.
TABLE V. COMPOSING TABLE OF SOM - K-MEANS CLUSTERS

\begin{tabular}{cccccc}
\hline \multirow{6}{*}{ Training institution version } & & \\
SOM cluster & K-Means cluster & Grade & Quantity & $\%$ & Total \\
02 & Cluster 1 & Gold & 12 & $100.00 \%$ & 12 \\
20 & Cluster 2 & Threshold & 86 & $100.00 \%$ & 86 \\
22 & Cluster 3 & Bronze & 46 & $65.71 \%$ & 70 \\
22 & Cluster 3 & Threshold & 24 & $34.29 \%$ & \\
00 & Cluster 4 & Bronze & 26 & $23.64 \%$ & \\
00 & Cluster 4 & Threshold & 45 & $40.91 \%$ & 110 \\
01 & Cluster 4 & Bronze & 39 & $35.45 \%$ & \\
02 & Cluster 5 & Silver & 18 & $13.85 \%$ & \\
02 & Cluster 5 & Bronze & 60 & $46.15 \%$ & 130 \\
12 & Cluster 5 & Bronze & 52 & $40.00 \%$ & \\
20 & Cluster 6 & Threshold & 60 & $98.36 \%$ & \\
21 & Cluster 6 & Threshold & 1 & $1.64 \%$ & 61 \\
11 & Cluster 7 & Bronze & 28 & $24.35 \%$ & \\
11 & Cluster 7 & Threshold & 16 & $13.91 \%$ & 115 \\
21 & Cluster 7 & Threshold & 71 & $61.74 \%$ & \\
10 & Cluster 8 & Threshold & 59 & $100.00 \%$ & 59 \\
20 & Cluster 9 & Threshold & 79 & $100.00 \%$ & 79 \\
\hline \multicolumn{5}{c}{ Data source: Sorted by this study } \\
\end{tabular}

3. In respect of the SOM clusters " 20,10 and 21 " and K-Means "Clusters 8, 9 and 6" falling in this area, because the composition in this area is solely "Threshold", it suggests that the organizations under review who fall in this area are relatively weak in the cognition and promotion of TTQS such that their levels of performance are lower than that by all TTQS promoting organizations.

The analytic results of the above-stated "verification and comparison by two-stage clustering" indicate that TTQS is able to assist the Taiwanese businesses and training institutions as a whole in the uplifting of train quality and human capital to a high extent and that by the consistence of the TTQS framework with the training system which the organization requires, the organization can incorporate a sound training system of high quality to further advance toward the achievement of the goal of continuous growth, self-breakthrough and sustainable operation.

\section{CONCLUSIONS}

The object of this study is to examine whether the TTQS evaluation system can bring effective and appropriate benefits to the human capital and education and training systems of businesses and training institutions as a whole in Taiwan, that is, to analyze the TTQS "review mechanism". Hence, courtesy of BEVT, the 2010 TTQS Review database was mined and the businesses and training institutions who have introduced TTQS were observed to see whether the indicators and specifications have all been internalized and carried out.

Research results are described below based on the analytic results from the research process built herein:

1. "Two-stage clustering" tests and compares the clustering results and verifies the consistence between grades for clusters. In the concept of similarity measurement by cluster analysis, the two-stage clustering of "SOM+K-Means" defined herein is employed to work on the 2010 TTQS Review database. First, data are clustered 
by SOM to obtain the initial solution (numbers of clusters) before the initial numbers of clusters are substituted in $\mathrm{K}$ Means to make step-two clustering on the nodes on the mapping chart. Such clustering results are used to verify the degree of consistence between the review operations by TTQS reviewers.

2. The "two-stage clustering" reveals that in the results in both "Business version" and "Training institution version", when the TTQS reviewers grade an organization for the levels of "Gold" and "Silver", it also suggests consistence in the grading with these two levels. In contrast, as with the grading for "Bronze" and "Threshold", less consistence is seen. The cause may be that the organizations that are graded for these two levels are less able to grasp the TTQS spirit and the requirements of the indicators in the introduction and execution of TTQS, such that they embody the TTQS with lower degree of completeness and thoroughness, which receives lower levels of grading from the reviewers.

Taiwan TrainQuali System has been able to positively and fast benefit the businesses and training institutions in Taiwan, assisting in the uplift of human capital and in the building of education and training system, further to draw the nation's attention to human resources and education and training, bringing up the nation's strength of human capital to be internationally competitive. The research findings are also provided as reference in continued exercise of TTQS in future for businesses and training institutions.

\section{ACKNOWLEDGMENT}

The authors would like to express the gratitude for the budget support from National Science Council on fitness test research program of TTQS (NSC 100-2410-H-167-010MY2) to make this research possible.

\section{REFERENCES}

[1] S.-T. Tzeng, "A Studying of Energy Performance Indicators In National Cheng Kung University," master thesis at the Graduate Institute of Resources Engineering, National Cheng Kung University, 2010.

[2] T. Kohonen, "The Self-Organizing Map. Proceedings of the IEEE,' 1990, pp.1464-1480.

[3] S.S. Abidi, and J. A Ong, "Data Mining Strategy for Inductive Data Clustering: Asynergy Between Self-Organising Neural Networks and K-Means Clustering Techniques," IEEE Transactions On Neural Networks, 2000, pp.568-573.

[4] J. Vesanto, and E. Alhoniemi, "Clustering of the Self-Organization Map,” IEEE Transactions On Neural Networks, 2000, pp.568-600.

[5] TTQS Project Office, TTQS Development strategy planning for 2010 to $2012,2009$.

[6] Y.-C. Yeh, "Application and practice of neural network models," $8^{\text {th }}$ ed., Taipei: Scholars Books, 2003. 